PROGRAMME

Journée d'étude

\section{NEUROSCIENCES PSYCHANALYSE}

\section{Un partenariat possible}

\section{journeedetuderph@gmail.com}

\section{S'inscrire : https://www.rphweb.fr}

Bulletin d'inscription à envoyer par courrier à Fairouz NEMRAOUI, 39 rue de Verdun 94220 Charenton-le-Pont

Nom

Prénom

Adresse

\section{Mail}

\section{6}

Organisateurs

Fairouz NEMRAOUI

Alexis POCHEZ

Matthieu JULIAN

Espace Vinci

25 rue des Jeûneurs 75002 Paris
PROGRAM M E

Journée d'étude

\section{NEUROSCIENCES PSYCHANALYSE}

Un partenariat possible

Les neurosciences s'intéressent au système nerveux, la psychanalyse étudie le psychisme humain. Deux disciplines différentes, un point commun : l'être parlant, doté d'un cerveau et d'un désir inconscient. Freud, inventeur de la psychanalyse, a lui-même commencé sa carrière en tant que neurologue. Aujourd'hui, pour être psychanalyste, il n'est pas nécessaire de passer par la formation neurologique. Pour autant, ces deux disciplines, neurosciences et psychanalyse, se rencontrent car toutes deux travaillent avec l'être parlant : par l'étude du cerveau pour les premiers, par celle du désir inconscient pour les seconds. Spécialistes du cerveau et psychanalystes peuvent être amenés à recevoir les mêmes patients, d'où l'importance de cultiver un travail en partenariat, respectueux des spécificités de chaque domaine d'expertise. Corps, organisme, chances de réveil, récupération, rechutes, résistances, plasticité cérébrale : à partir des interventions de médecins et des cliniciens membres du RPH, nous verrons au cours de cette journée d'études comment psychanalyse et neurosciences peuvent s'allier pour une meilleure prise en charge du patient. Cette journée s'inscrit dans l'esprit du travail en partenariat, par lequel chaque spécialiste travaille de concert avec l'autre.

\section{NEUROSCIENCES \& PSYCHANALYSE}

Un partenariat possible

\author{
PROGRAMME \\ Journée d'étude
}

Samedi 30 janvier 2021 de $9 h$ à $17 h$ 
P R OG RAMM E

Journée d'étude

\section{NEUROSCIENCES}

\section{PSYCHANALYSE}

\section{Un partenariat possible}

\section{MATINÉE}

$9 h 30$ - 9h45 : Ouverture

Docteur Fernando de Amorim, psychanalyste

09h45 - 10h05 : Professeur Frédéric Clarençon, neuroradiologue : Neuroradiologie interventionnelle un peu plus qu'une histoire de tuyaux....

10h05 - 10h20 : Fairouz Nemraoui, psychothérapeute, doctorante : La clinique du partenariat

10h20 - 10h40 : Docteur Lydia Chougar, neuroradiologue : La neuroimagerie,

un moyen d'exploration du cerveau

$$
\begin{gathered}
10 \mathrm{~h} 40 \text { - 11h10 : Discussion } \\
11 \mathrm{~h} 10 \text { - 11h30 : Pause }
\end{gathered}
$$

11h30 - 11h45 : Marine Bontemps, psychothérapeute, doctorante: Ce que la psychanalyse gagne à s'inscrire dans la voie de la science

11h45 - 12h05 : Professeur Damien Galanaud, neuroradiologue : Quels sont les intérêts de réaliser une imagerie pronostique pour les patients dans le coma?

\section{2h05 - 12h30 : Discussion}

Discutant de la matinée : Ouarda Ferlicot, psychanalyste

\section{NEUROSCIENCES PSYCHANALYSE}

Un partenariat possible

\section{APRĖS-MIDI}

14h30 - 14h50 : Docteur Elise Mathy, onco-endocrinologue : Se réapproprier et aimer à nouveau son corps après un handicap acquis

14h50 - 15h05 : Alexis Pochez, psychothérapeute, doctorant : Neurosciences et temps d'écran argument pour la castration symbolique parentale

\section{5h05 - 15h35 : Discussion $15 h 35-15 h 45$ : Pause}

15h45 - 16h05 : Docteur Eric Gibert, rhumatologue : Intérêt de la pratique de l'hypnose dans les gestes interventionnels en neuroradiologie

16h05 - 16h25 : Docteur Patrick Belamich, psychiatre : Neurosciences, psychanalyse et CMPP

16h25 - 17h : Discussion et mot de la fin

Discutant de l'après-midi :

Docteur Julie Mortimore, psychothérapeute

Modérateur de la journée :

Matthieu Julian, psychothérapeute, doctorant
NEUROSCIENCES PSYCHANALYSE

Un partenariat possible

\section{TARIFS}

$40 €$ et $15 €$ pour les étudiants

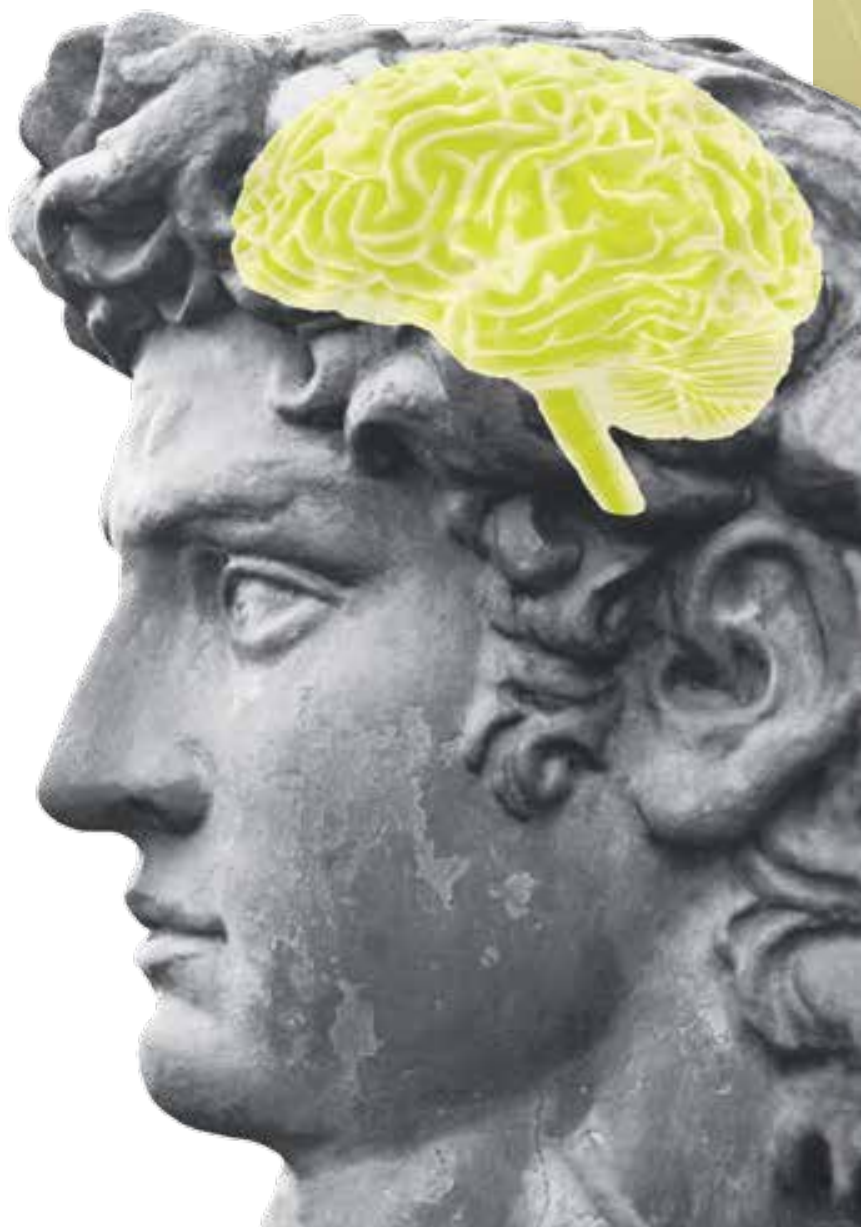

